\title{
Determination of phase equilibria in confined systems by open pore cell Monte Carlo method.
}

\section{$\operatorname{AUTHOR}(\mathrm{S})$ :}

Miyahara, Minoru T; Tanaka, Hideki

\section{CITATION:}

Miyahara, Minoru T ...[et al]. Determination of phase equilibria in confined systems by open pore cell Monte Carlo method.. The Journal of chemical physics 2013, 138(8): 084709.

ISSUE DATE:

2013-02-28

URL:

http://hdl.handle.net/2433/173108

RIGHT:

(C) 2013 American Institute of Physics 


\section{AIP $\begin{gathered}\text { medoumalo } \\ \text { chemical Physics }\end{gathered}$}

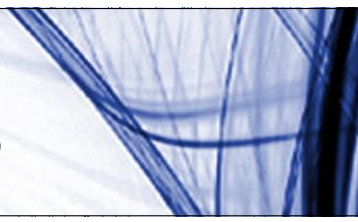

\section{Determination of phase equilibria in confined systems by open pore cell Monte Carlo method}

Minoru T. Miyahara and Hideki Tanaka

Citation: J. Chem. Phys. 138, 084709 (2013); doi: 10.1063/1.4792715

View online: http://dx.doi.org/10.1063/1.4792715

View Table of Contents: http://jcp.aip.org/resource/1/JCPSA6/v138/i8

Published by the American Institute of Physics.

\section{Additional information on J. Chem. Phys.}

Journal Homepage: http://jcp.aip.org/

Journal Information: http://jcp.aip.org/about/about_the_journal

Top downloads: http://jcp.aip.org/features/most_downloaded

Information for Authors: http://jcp.aip.org/authors

\section{ADVERTISEMENT}

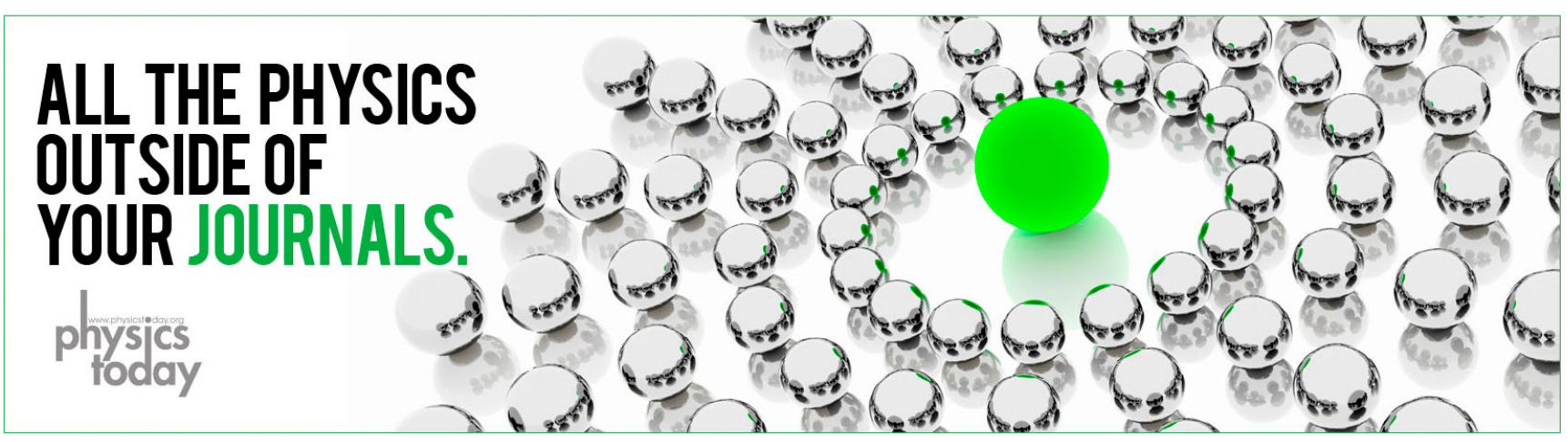




\title{
Determination of phase equilibria in confined systems by open pore cell Monte Carlo method
}

\author{
Minoru T. Miyahara ${ }^{\text {a) }}$ and Hideki Tanaka \\ Department of Chemical Engineering, Kyoto University, Katsura, Nishikyo, Kyoto 615-8510, Japan
}

(Received 5 November 2012; accepted 6 February 2013; published online 28 February 2013)

\begin{abstract}
We present a modification of the molecular dynamics simulation method with a unit pore cell with imaginary gas phase [M. Miyahara, T. Yoshioka, and M. Okazaki, J. Chem. Phys. 106, 8124 (1997)] designed for determination of phase equilibria in nanopores. This new method is based on a Monte Carlo technique and it combines the pore cell, opened to the imaginary gas phase (open pore cell), with a gas cell to measure the equilibrium chemical potential of the confined system. The most striking feature of our new method is that the confined system is steadily led to a thermodynamically stable state by forming concave menisci in the open pore cell. This feature of the open pore cell makes it possible to obtain the equilibrium chemical potential with only a single simulation run, unlike existing simulation methods, which need a number of additional runs. We apply the method to evaluate the equilibrium chemical potentials of confined nitrogen in carbon slit pores and silica cylindrical pores at $77 \mathrm{~K}$, and show that the results are in good agreement with those obtained by two conventional thermodynamic integration methods. Moreover, we also show that the proposed method can be particularly useful for determining vapor-liquid and vapor-solid coexistence curves and the triple point of the confined system. (C) 2013 American Institute of Physics. [http://dx.doi.org/10.1063/1.4792715]
\end{abstract}

\section{INTRODUCTION}

The determination of chemical potential is indispensable when studying the thermodynamic equilibrium of a confined fluid in a small pore through molecular simulations. The development of the method for evaluating the chemical potential of such a confined inhomogeneous system has a long history since the seminal work of Widom. ${ }^{1}$ However, it remains a subject of continuing interest, especially for the determination of the equilibrium phase transition between vaporlike and liquid-like states. The reason for this interest is that the vapor-liquid transition of the confined fluid involves multiple stable and metastable states. Therefore, the free energy of the system has to be calculated numerically (e.g., thermodynamic integration as discussed below) to locate the thermodynamic coexistence point or certain constraints have to be imposed to scan a limited area of the system configuration space in molecular simulations.

To the best of our knowledge, the test particle insertion method of Widom was first applied to investigate the vaporliquid coexistence of the confined fluid by Heffelfinger et al. ${ }^{2}$ In their pioneering work, a canonical molecular dynamics (MD) simulation was used to study the vapor-liquid coexistence system of a Lennard-Jones (LJ) fluid separated by hemispherical menisci in a cylindrical pore, and the thermodynamic equilibrium of the capillary liquid was confirmed by the equality of the chemical potentials in the vapor and liquid regions. Since then, the Widom method has been successfully employed in many studies of confined systems. ${ }^{3-8}$ However, the application of the Widom method can result in large uncertainties, which result from a piling up of the test particle

\footnotetext{
a) Author to whom correspondence should be addressed. Electronic mail: miyahara@cheme.kyoto-u.ac.jp.
}

insertions having a negligibly small Boltzmann factor in pore fluids with high density. ${ }^{9}$

For adsorption studies, a natural ensemble that can be used is the grand canonical ensemble, and, therefore, the grand canonical Monte Carlo (GCMC) method is a powerful tool to study the vapor-liquid transition of the confined fluid. However, at subcritical temperatures, the GCMC method exhibits a hysteresis loop formed by discontinuous condensation and evaporation branches for a pore employing periodic boundary conditions so that the resulting pore structure is infinite in length. This is because of extended metastability on the vapor-like and liquid-like states, and therefore, in such a case, the GCMC method is not capable of determining the equilibrium phase transition. Peterson and Gubbins proposed that the equilibrium chemical potential could be found by calculating the grand potential via thermodynamic integration, ${ }^{10}$ and their method has been used as a standard method to determine the phase coexistence in confined systems. ${ }^{5,11,12}$ Their method, however, requires additional intense GCMC simulations to circumvent a discontinuity due to the spontaneous condensation and to perform thermodynamic integration along a continuous trajectory. Papadopoulou et al. studied the adsorption hysteresis for a pore with open ends (hard wall vapor reservoirs) by using the GCMC method, and found that the metastability on the liquid-like state is removed due to the symmetry breaking and, thus, the pore emptying occurs at thermodynamic equilibrium. ${ }^{13}$ Their method is considered to be useful for determining the equilibrium chemical potential; however, to locate the equilibrium phase transition with high accuracy, GCMC simulations at a number of chemical potentials should be required.

The pore-pore Gibbs ensemble Monte Carlo (GEMC) method, proposed by Panagiotopoulos, ${ }^{14}$ is also a direct 
approach for determining the vapor-liquid coexistence of the confined fluid. The method yields vapor and liquid phases in two pore cells, which are in phase equilibrium, by allowing for both mass and volume exchange between the pore cells. Although the pore-pore GEMC method has been extensively employed for studies in phase equilibria of confined fluids, ${ }^{15-19}$ the equilibrium chemical potential $\mu_{\mathrm{eq}}$ cannot be easily obtained. The $\mu_{\mathrm{eq}}$ value then has to be estimated by the Widom method, which is problematic for a high density phase, or by interpolating the corresponding GCMC adsorption isotherm with the equilibrium vapor and liquid densities obtained from the GEMC simulation. Moreover, Neimark and Vishnyakov pointed out that, when the pore-pore GEMC method was applied to a system with strongly attractive pore walls, the resulting coexistence point was sensitive to the initial configurations, which from the suppression of the volume exchange trial owing to the formation of dense contact layer adjacent to the pore wall. ${ }^{20}$

In 1997, Miyahara et al. developed a unique MD simulation pore cell, which is opened to a bulk vapor phase (open pore cell MD). ${ }^{21}$ The thermodynamic equilibrium of the confined fluid is easily achieved because the open nature of the pore cell introduces the symmetry breaking and therefore leads to ease in holding stable menisci within the pore cell. Another benefit of the open pore cell MD method is that the equilibrium vapor pressure is easily determined by simply counting the frequency of particles reaching the vapor phase out of the attractive pore space, in contrast to the MD method by Heffelfinger et al. ${ }^{2}$ and the GCMC method by Papadopoulou et al. ${ }^{13}$ The robust property of the open pore cell MD method has contributed considerably to studies seeking to determine the phase diagram of the $\mathrm{LJ}$ fluid, ${ }^{22-26}$ and to the development of a new capillary condensation model for nanopores. ${ }^{27-30}$ However, in some cases, at low temperatures and associated low equilibrium vapor pressures, this method has a drawback in that the computational cost becomes large in allowing for a sufficient number of particles to reach the vapor phase.

As an alternative method for determining the equilibrium phase transition by thermodynamic integration, Neimark and Vishnyakov proposed the gauge cell method in $2000 .{ }^{20}$ Their method is, in principle, similar to the pore-fluid GEMC method. ${ }^{14}$ The MC simulation is performed simultaneously in two cells (pore cell and gas cell), which can exchange particles in such a way that the total number of particles remains fixed. The total system composed of the two cells is in the canonical ensemble, and the cell volumes are kept unchanged, in contrast to the pore-pore GEMC method. ${ }^{14}$ The gas cell then serves as a "gauge" to measure the chemical potential of the system, which is where the name of this method comes from. The chemical potential of the system is calculated by the Johnson-Zollweg-Gubbins equation of state ${ }^{31}$ using the ensemble average of the LJ fluid density in the gas cell. In the modified version of the gauge cell method, the fluid in the gas cell is treated as ideal (mesoscopic canonical ensemble). ${ }^{32}$ The key feature of the gauge cell method is that the limited volume of the gas cell constrains density fluctuations in the pore cell, and allows the adsorbed phase to be a metastable state and, moreover, a thermodynamically unstable state. Namely, the method enables the construction of a van der Waals-type sigmoid adsorption isotherm. The phase coexistence is thus determined as the points of equal grand potential, which satisfies the Maxwell rule of equal areas, by the thermodynamic integration of the sigmoid adsorption isotherm. Because of the excellent suitability of this method for the confined inhomogeneous system, the gauge cell method has been used for studies of vapor-liquid coexistences in various systems. ${ }^{33-44}$ However, according to Vishnyakov and Neimark, ${ }^{33}$ the method is not suited for a system at low temperatures such that the confined fluid enters into a glass or crystalline state, because very few attempts of particle exchange are successful, making it difficult to reach equilibrium. Moreover, significant computational effort is required for optimizing the size of the gas cell and to obtain a continuous sigmoid adsorption isotherm required for precise thermodynamic integration.

Recently, Liu et al. proposed a unique method to determine the equilibrium phase transition of the confined fluid. ${ }^{45,46}$ Their method is called the "mid-density scheme" and is a combination of the canonical MC and GCMC methods to locate the equilibrium chemical potential within a hysteresis loop obtained by the standard GCMC method. They constructed a configuration with mid-density, which is the average density between the low-density and high-density states at a given chemical potential within the hysteresis loop, and they then performed the canonical MC simulation to fully relax the system. Next, the GCMC simulation was started with the equilibrated configuration at the same chemical potential. The system will evolve to a high-density state if the fluid density in the mid-density state is larger than that of a transient state separating the low-density and high-density states, whereas the contrary will happen when the mid-density is lower than that of the transient state. Therefore, the threshold chemical potential can be determined by performing the GCMC simulations with the mid-density configurations at several chemical potentials within the hysteresis loop. The mid-density method is easy and useful for a rough estimate of the equilibrium chemical potential. However, the number of significant digits of the obtained equilibrium chemical potential is small because this method follows a discrete approach.

In this work, we develop a Monte Carlo version of the open pore cell MD method ${ }^{21}$ designed for determination of phase equilibria in nanopores. The practical effectiveness of our open pore cell Monte Carlo (MC) method is verified by comparing with the Peterson and Gubbins method ${ }^{10}$ and the gauge cell method. ${ }^{20}$ In addition, we demonstrate an excellent property of the open pore cell MC method for obtaining the vapor-solid coexistence curve in a narrow pore at which equilibrium vapor pressure is extremely low.

\section{METHODS}

\section{A. Peterson-Gubbins (PG) method}

Typical adsorption and desorption isotherms of the $\mathrm{LJ}$ fluid in a nanopore by the GCMC method are shown in Fig. 1 (lines $A D J K$ and $K H B A$ ). The temperature of the system is $T_{1}$, which is below the capillary critical point. The 


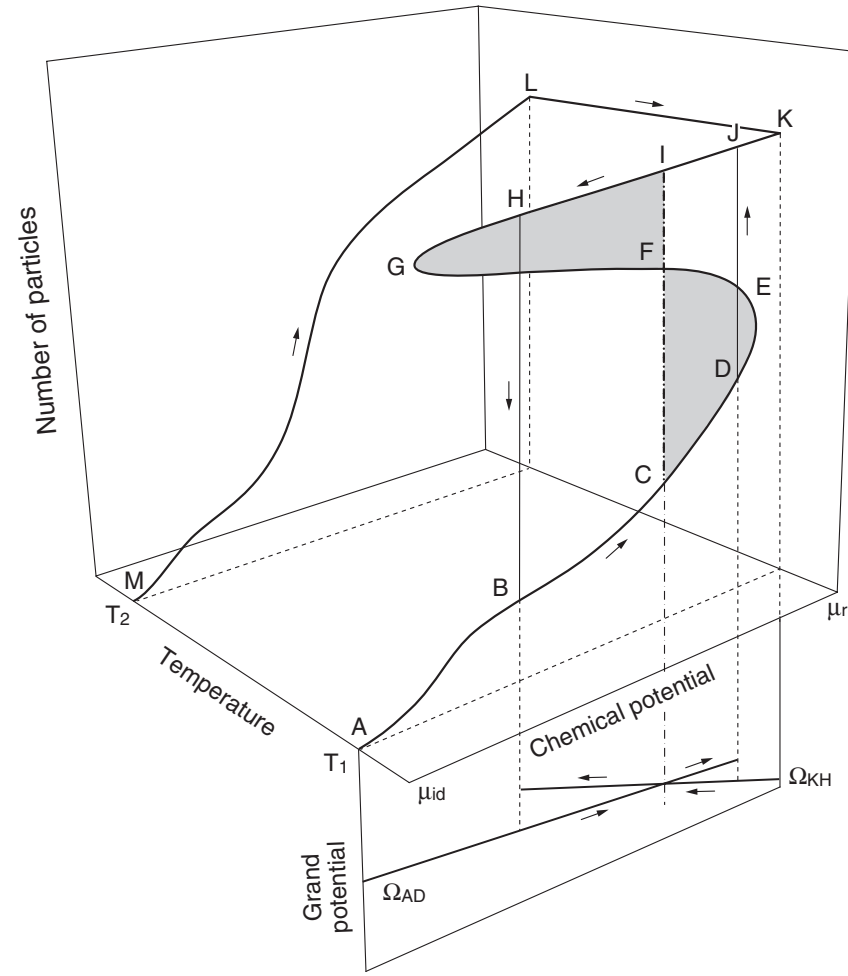

FIG. 1. Explanatory diagram showing the paths considered in the two thermodynamic integration methods (PG and GC methods) for determining the vapor-liquid coexistence in a pore at subcritical temperature $T_{1}$. The vertical line $C I$ represents the equilibrium vapor-liquid coexistence, where the grand potentials, $\Omega_{A D}$ and $\Omega_{K H}$, are equal. The shaded areas, $C D E F$ and $F G H I$, are equal according to the Maxwell rule. Other details are described in the text.

adsorption isotherm is discontinuous because of a spontaneous condensation from a vapor-like state (point $D$ ) to a liquid-like state (point $J$ ). Likewise, the desorption isotherm contains a spontaneous evaporation from point $H$ to point $B$. The lines $C D$ and $I H$ correspond to metastable states, and the limiting metastabilities are dominated by energetic barriers separating the vapor-like and liquid-like states, which the system should overcome. The vertical line $C I$ represents the vapor-liquid coexistence (equilibrium chemical potential $\mu_{\mathrm{eq}}$ ), and the grand thermodynamic potentials at the points $C$ and $I$ are equal. However, the grand thermodynamic potential is not a direct output of the GCMC simulation. According to Peterson and Gubbins,${ }^{10}$ the grand thermodynamic potential, $\Omega_{A D}$, along the continuous adsorption branch (line $A D$ ) is calculated by integrating the Gibbs adsorption isotherm, $N_{A D}$ :

$$
\Omega_{A D}\left(\mu, T_{1}\right)=\Omega_{A}\left(\mu_{\mathrm{id}}, T_{1}\right)-\int_{\mu_{\mathrm{id}}}^{\mu} N_{A D}\left(\mu^{\prime}, T_{1}\right) d \mu^{\prime},
$$

where $\mu$ is a given chemical potential. The first term in the right-hand side is the grand thermodynamic potential at chemical potential $\mu_{\mathrm{id}}$, which is low enough so that the adsorption amount $N_{A}$ at point $A$ is essentially the ideal gas value:

$$
\Omega_{A}\left(\mu_{\mathrm{id}}, T_{1}\right)=-k T_{1} N_{A}\left(\mu_{\mathrm{id}}, T_{1}\right),
$$

where $k$ is the Boltzmann constant. The grand thermodynamic potential $\Omega_{K H}$ along the desorption branch (line $K H$ ) is calculated by integrating the Gibbs adsorption isotherm $N_{K H}$ from point $K$ at chemical potential $\mu_{\mathrm{r}}$. Point $K$ can be arbitrarily chosen if $\mu_{\mathrm{eq}}<\mu_{\mathrm{r}}$. The $\Omega_{K}\left(\mu_{\mathrm{r}}, T_{1}\right)$ value is obtained by integration along two reversible paths, namely, a supercritical adsorption isotherm $N_{M L}$ at temperature $T_{2}$ (line $M L$ ) and a path at the constant chemical potential $\mu_{\mathrm{r}}$ (line $L K$ ), which connects the two isotherms at $T_{1}$ and $T_{2}$. The integration of the supercritical adsorption isotherm from $\mu_{\mathrm{id}}$ (point $M$ ) to $\mu_{\mathrm{r}}$ (point $L$ ) is expressed as

$$
\Omega_{L}\left(\mu_{\mathrm{r}}, T_{2}\right)=-k T_{2} N_{M}\left(\mu_{\mathrm{id}}, T_{2}\right)-\int_{\mu_{\mathrm{id}}}^{\mu_{\mathrm{r}}} N_{M L}\left(\mu, T_{2}\right) d \mu,
$$

and then, the integration along the path $L K$ at the constant chemical potential $\mu_{\mathrm{r}}$ from $T_{2}$ to $T_{1}$ is expressed as

$$
\begin{aligned}
\Omega_{K}\left(\mu_{\mathrm{r}}, T_{1}\right)= & \Omega_{L}\left(\mu_{\mathrm{r}}, T_{2}\right) T_{1} / T_{2}+T_{1} \int_{1 / T_{2}}^{1 / T_{1}}\left[E_{L K}\left(\mu_{\mathrm{r}}, T\right)\right. \\
& \left.-N_{L K}\left(\mu_{\mathrm{r}}, T\right) \mu_{\mathrm{r}}\right] d(1 / T),
\end{aligned}
$$

where $N_{L K}$ is the number of particles and $E_{L K}$ is the sum of the potential energy and the kinetic energy ( $3 N k T / 2)$ along the line $L K$. Therefore, the grand thermodynamic potential $\Omega_{K H}$ is obtained as

$$
\Omega_{K H}\left(\mu, T_{1}\right)=\Omega_{K}\left(\mu_{\mathrm{r}}, T_{1}\right)-\int_{\mu_{\mathrm{r}}}^{\mu} N_{K H}\left(\mu^{\prime}, T_{1}\right) d \mu^{\prime} .
$$

Finally, the true phase equilibrium is determined as the point of intersection between the grand thermodynamic potentials, $\Omega_{A D}$ and $\Omega_{K H}$ (see Fig. 1).

\section{B. Gauge cell (GC) method}

A typical van der Waals-type sigmoid adsorption isotherm obtained by the gauge cell method is also shown in Fig. 1 (continuous line from point $A$ to point $K$ ). The gauge cell method can give the limits of the vapor-like spinodal (point $E$, chemical potential $\mu_{\mathrm{vs}}$ ) and liquidlike spinodal (point $G$, chemical potential $\mu_{\mathrm{ls}}$ ), and unstable states (line $E G$ ). The grand thermodynamic potential, $\Omega_{C}\left(\mu_{\mathrm{eq}}, T_{1}\right)$, at point $C$ can be obtained by integrating, as in Eq. (1), the adsorption isotherm as

$$
\Omega_{C}\left(\mu_{\mathrm{eq}}, T_{1}\right)=\Omega_{A}\left(\mu_{\mathrm{id}}, T_{1}\right)-\int_{\mu_{\mathrm{id}}}^{\mu_{\mathrm{eq}}} N_{A C}\left(\mu, T_{1}\right) d \mu .
$$

Similarly, the grand thermodynamic potential, $\Omega_{I}\left(\mu_{\mathrm{eq}}, T_{1}\right)$, at point $I$ can be calculated as

$$
\begin{aligned}
\Omega_{I}\left(\mu_{\mathrm{eq}}, T_{1}\right)= & \Omega_{C}\left(\mu_{\mathrm{eq}}, T_{1}\right)-\int_{\mu_{\mathrm{eq}}}^{\mu_{\mathrm{vs}}} N_{C E}\left(\mu, T_{1}\right) d \mu \\
& -\int_{\mu_{\mathrm{vs}}}^{\mu_{\mathrm{ls}}} N_{E G}\left(\mu, T_{1}\right) d \mu-\int_{\mu_{\mathrm{ls}}}^{\mu_{\mathrm{eq}}} N_{G I}\left(\mu, T_{1}\right) d \mu .
\end{aligned}
$$

The vapor-liquid coexistence condition, $\Omega_{I}\left(\mu_{\text {eq }}, T_{1}\right)$ $=\Omega_{C}\left(\mu_{\mathrm{eq}}, T_{1}\right)$, gives the Maxwell rule that the areas $C D E F$ and $F G H I$ are equal:

$$
\begin{gathered}
\int_{\mu_{\mathrm{ls}}}^{\mu_{\mathrm{vs}}} N_{E G}\left(\mu, T_{1}\right) d \mu-\int_{\mu_{\mathrm{eq}}}^{\mu_{\mathrm{vs}}} N_{C E}\left(\mu, T_{1}\right) d \mu \\
-\int_{\mu_{\mathrm{ls}}}^{\mu_{\mathrm{eq}}} N_{G I}\left(\mu, T_{1}\right) d \mu=0 .
\end{gathered}
$$



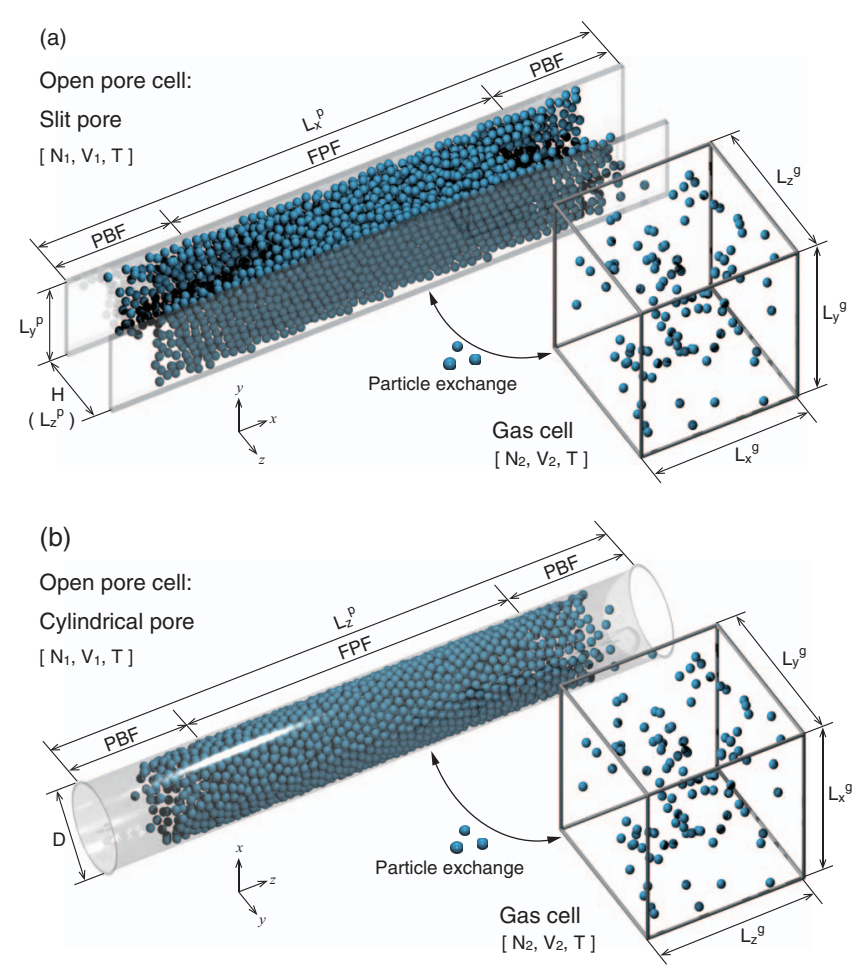

FIG. 2. Schematic representations of the simulation cells used in the OC method for (a) slit pore and (b) cylindrical pore. The open pore cells are composed of two potential fields: the FPF where a full interaction potential is exerted by the pore wall, and the PBF. The interaction potential in the PBF is linearly attenuated from the full potential value to zero. The open pore cell and gas cells exchange particles under the condition that the number of particles, $N_{1}+N_{2}$, is constant.

\section{Open pore cell Monte Carlo (OC) method}

The aforementioned two methods are both based on thermodynamic integration, and thus the estimated equilibrium chemical potential may be fatally affected by not only statistical errors in the simulated isotherm but also errors in the numerical integration of the isotherm. Therefore, we employ the pore cell with a "potential buffering field," which was developed for determining phase equilibria in nanopores by MD simulation, ${ }^{21}$ and combine it with a gas cell to measure the chemical potential of the system similar to the gauge cell method. Our system with the two simulation cells developed for this study is shown in Fig. 2. The pore cell is composed of two potential fields: the full potential field (FPF), where a "full" interaction potential is exerted by the pore wall, and the potential buffering field (PBF). The interaction potential in the PBF is continuously and linearly attenuated from the full potential value to zero, so that both ends of the pore cell are considered to be connected to an imaginary gas phase. The name of our proposed method "open pore cell" stems from the characteristics of the pore cell. The most striking feature of the open pore cell with the PBF is that the system is steadily led to a thermodynamically stable state by forming concave menisci, when the system contains a sufficient number of particles. This feature enables estimation of the equilibrium chemical potential with only a single simulation run by combining with the gas cell. As stated in the Introduction, Papadopoulou et al. attached the hard wall vapor reservoirs to the pore ends in their GCMC simulations, differently from our open pore cell. ${ }^{13}$ The discontinuity in the potential field of the pore model will be likely to perturb the structure of the concave meniscus of the confined liquid, which may give a different equilibrium chemical potential with that for a corresponding pore model employing periodic boundary conditions. Another worry for the discontinuity would be that the pore fluid, especially those particles in the first contacting layer, may be subjected to an artificial dense-packed state brought by the finite size of the pore potential field with sharp edges. One more advantage of our open pore cell is that the border between the FPF and the PBF can be clearly defined, which is important to obtain an exact equilibrium chemical potential as described in Sec. IV A, differently from the recent atomistic finite pore models with open ends. ${ }^{47,48}$ Then, originally, in the MD version of the open pore cell method, the equilibrium vapor pressure was determined by counting the number of molecules reaching the border plane at the end of the pore cell. However, when the pore size is small and/or the system temperature is low, the frequency of particle counting was decreased, and therefore, a long run of the MD simulation was required to obtain good statistics. Our proposed method can overcome these disadvantages by adjusting the size of the gas cell. The choice of the size of the gas cell is straightforward unlike the GC method, in which it must be chosen to meet a particular condition (described in Sec. III B).

\section{SIMULATION DETAILS}

To verify the usefulness of the open pore cell method over the PG and GC methods, we applied each of the three methods to evaluate the equilibrium chemical potentials of confined nitrogen in carbon slit pores and silica cylindrical pores at $77 \mathrm{~K}$. The LJ potential with the parameters, $\sigma_{\mathrm{ff}}=0.3615 \mathrm{~nm}$ and $\varepsilon_{\mathrm{ff}} / k=101.5 \mathrm{~K}$ ( $k$ is the Boltzmann constant), and a cutoff radius of $5 \sigma_{\mathrm{ff}}$ were used for the fluid-fluid interactions of nitrogen. ${ }^{49}$

The carbon slit pores were modeled by two graphite slabs with a pore width $H$, where $H$ is defined as the distance between the graphite slabs as measured from the carbon centers at the surfaces. The solid-fluid interaction potential between a $\mathrm{LJ}$ nitrogen and one graphite slab is represented by the Steele 10-4-3 potential:

$$
\begin{aligned}
\phi(z)= & 2 \pi \rho_{\mathrm{s}} \varepsilon_{\mathrm{sf}} \sigma_{\mathrm{sf}}^{2} \Delta\left[\frac{2}{5}\left(\frac{\sigma_{\mathrm{sf}}}{z}\right)^{10}-\left(\frac{\sigma_{\mathrm{sf}}}{z}\right)^{4}\right. \\
& \left.-\frac{\sigma_{\mathrm{sf}}^{4}}{3 \Delta(0.61 \Delta+z)^{3}}\right],
\end{aligned}
$$

where $\rho_{\mathrm{S}}$ is the number density of carbon atoms in graphite, $\Delta$ is the interlayer spacing of graphene sheets, and $\sigma_{\text {sf }}$ and $\varepsilon_{\text {sf }}$ are the $\mathrm{LJ}$ parameters for the solid-fluid interaction. The parameters used in this study are tabulated in Table I. ${ }^{49}$ The total potential for a LJ nitrogen in the carbon slit pore is obtained by adding the contributions from each wall as follows:

$$
\Phi(z)=\phi(z)+\phi(H-z) .
$$

Periodic boundary conditions in the $x$ and $y$ directions were applied, and the lateral dimensions of the simulation cell were 
TABLE I. Parameters for the intermolecular potentials.

\begin{tabular}{|c|c|c|c|c|c|c|c|}
\hline \multicolumn{2}{|c|}{ Fluid-fluid } & \multicolumn{6}{|c|}{ Solid-fluid } \\
\hline$\sigma_{\mathrm{ff}}(\mathrm{nm})$ & $\varepsilon_{\mathrm{ff}} / k(\mathrm{~K})$ & $\sigma_{\mathrm{sf}}(\mathrm{nm})^{\mathrm{a}}$ & $\varepsilon_{\mathrm{sf}} / k(\mathrm{~K})^{\mathrm{a}}$ & $\rho_{\mathrm{s}}\left(\mathrm{nm}^{-3}\right)^{\mathrm{a}}$ & $\sigma_{\mathrm{sf}}(\mathrm{nm})^{\mathrm{b}}$ & $\varepsilon_{\mathrm{sf}} / k(\mathrm{~K})^{\mathrm{b}}$ & $\rho_{\mathrm{s}}\left(\mathrm{nm}^{-2}\right)^{\mathrm{b}}$ \\
\hline 0.3615 & 101.5 & 0.3508 & 53.31 & 114 & 0.317 & 147.3 & 15.3 \\
\hline
\end{tabular}

${ }^{\text {a }}$ Parameters for the carbon slit pore.

${ }^{\mathrm{b}}$ Parameters for the silica cylindrical pore.

set as $L_{x}{ }^{\mathrm{p}}=L_{y}{ }^{\mathrm{p}}=10 \sigma_{\mathrm{ff}}$ in the case of the PG and GC methods. Those for the OC method are described in Sec. III C. We chose three pore sizes of $H / \sigma_{\mathrm{ff}}=H^{*}=7,10$, and 13 for comparison.

The interaction potential of a LJ nitrogen inside the silica cylindrical pore with a single structureless layer of oxygen atoms is given by ${ }^{50}$

$$
\begin{aligned}
\Phi(s, R)= & \pi^{2} \rho_{\mathrm{s}} \varepsilon_{\mathrm{sf}} \sigma_{\mathrm{sf}}^{2}\left[\frac{63}{32} \frac{F\left(-4.5,-4.5,1.0 ; \xi^{2}\right)}{\left[R^{*}\left(1-\xi^{2}\right)\right]^{10}}\right. \\
& \left.-3 \frac{F\left(-1.5,-1.5,1.0 ; \xi^{2}\right)}{\left[R^{*}\left(1-\xi^{2}\right)\right]^{4}}\right],
\end{aligned}
$$

where, $\xi=R / s, s$ is the distance between a LJ nitrogen and the center of the cylindrical pore, $R$ is the radius of the cylindrical pore, $\rho_{\mathrm{s}}$ is the number density of oxygen atoms, $R^{*}$ $=R / \sigma_{\text {sf }}$, and $F(a, b, c ; d)$ is the hypergeometric function. The parameters used are listed in Table I. ${ }^{49}$ We define the pore diameter $(D=2 R)$ as the distance between the oxygen centers, and we adapted three pore sizes of $D / \sigma_{\mathrm{ff}}=D^{*}=10,13$, and 16 for comparison. A periodic boundary condition was employed only in the $z$ direction, and the length of the simulation cell was set to be $L_{z}{ }^{\mathrm{p}}=10 \sigma_{\mathrm{ff}}$ in the case of the PG and $\mathrm{GC}$ methods. The settings for the $\mathrm{OC}$ method are described in Sec. III C.

\section{A. PG method}

By using the GCMC method, we generated an adsorption-desorption isotherm at $77 \mathrm{~K}$, a supercritical adsorption isotherm at $140 \mathrm{~K}$, and a path at the constant chemical potential $\mu_{r}$, which connects the two isotherms. To determine the standard error of the equilibrium chemical potential obtained by the PG method, we constructed five different paths according to Neimark and Vishnyakov, ${ }^{20}$ namely, $\mu_{r} / \varepsilon_{\mathrm{ff}}=\mu_{r}{ }^{*}=-9.930,-9.781,-9.674,-9.572$, and -9.483 for a slit pore of $H^{*}=7$. At each $\mu_{r}{ }^{*}$ value, we performed the GCMC simulations at five different temperatures: $130 \mathrm{~K}, 120 \mathrm{~K}, 110 \mathrm{~K}, 100 \mathrm{~K}$, and $90 \mathrm{~K}$. In the GCMC simulations, three trial movements, namely, displacement, creation, and deletion, were performed with the same probabilities. The system was equilibrated for $2.5 \times 10^{7}$ Monte Carlo steps, after which data were collected for another $2.5 \times 10^{7}$ steps. The length of the Markov chain of $2.5 \times 10^{7}$ steps corresponds to more than $1.6 \times 10^{4}$ configurations per particle for all the cases investigated in this study. The final configuration was used as the initial state for the next simulation run.

\section{B. GC method}

In the GC method, two types of trial movements were performed: particle displacement in both the pore cell and the gas cell, and particle exchange between the two cells. The acceptance rules are the same as those of the Gibbs ensemble simulation $^{9,14}$ according to Vishnyakov and Neimark. ${ }^{33}$ The probabilities of the displacement and the particle exchange were set to be equal. Since the potential energy of a trial particle inserted into the gas cell corresponds to the test particle energy of the Widom insertion method, the chemical potential of the system can be obtained without any additional cost by ${ }^{9}$

$$
\mu=-\frac{1}{k T} \ln \frac{1}{\Lambda^{3}}\left\langle\frac{V_{\mathrm{g}}}{N_{\mathrm{g}}+1} \exp \left(-\frac{\Delta u_{\mathrm{g}}}{k T}\right)\right\rangle_{\text {gas cell }},
$$

where $\Lambda$ is the thermal de Broglie wavelength, $V_{\mathrm{g}}$ is the volume of the gas cell, $N_{\mathrm{g}}$ is the number of particles in the gas cell, and $\Delta u_{\mathrm{g}}$ is the potential energy of a test particle. The dimensions of the gas cell were set to be $L_{x}{ }^{g}=L_{y}{ }^{g}=L_{z}{ }^{g}=40$ $\sigma_{\text {ff }}$, and periodic boundary conditions in the three directions were applied. The size of the gas cell was chosen to meet the condition $^{20}$

$$
\frac{V_{\mathrm{g}}}{V_{\mathrm{p}}}<\frac{k T}{\rho_{\mathrm{g}}}\left|\frac{\partial \rho_{\mathrm{p}}}{\partial \mu}\right|,
$$

where $V_{\mathrm{p}}$ is the volume of the pore cell, $\rho_{\mathrm{p}}$ and $\rho_{\mathrm{g}}$ are the fluid densities in the pore cell and gas cell. Provided Eq. (13) is satisfied, an unstable state with a negative compressibility is stabilized in the pore cell.

The system was equilibrated for $1 \times 10^{4}$ MC steps/ particle, after which the average properties of the system were collected for another $2 \times 10^{7}$ steps. To obtain a sigmoid adsorption isotherm for a target pore, the final configurations were used as the initial configurations for the next simulation run after a given number of particles were added into the gas cell. The equilibrium chemical potential was evaluated from the sigmoid adsorption isotherm by using Eq. (8). We performed simulations of $2 \times 10^{7} \mathrm{MC}$ steps from the final configurations of each point of the sigmoid adsorption isotherm, and repeated it four times. Thus, we generated five sigmoid adsorption isotherms for a target pore to determine the standard error of the equilibrium chemical potential obtained by the GC method.

\section{OC method}

Figures 2(a) and 2(b) show the open pore cells for the carbon slit and silica cylindrical pore geometries. The length of the open pore cell of the carbon slit pore was set to be $L_{x} \mathrm{p}=70 \sigma_{\text {ff. }}$. The FPF was placed at the center along the $x$ 
direction and its length was $40 \sigma_{\mathrm{ff}}$. The solid-fluid interaction potential in the FPF (Eq. (10)) was linearly attenuated from the full potential value to zero in the two PBFs $\left(15 \sigma_{\mathrm{ff}}\right.$ in length) along the $x$ direction. The length of the open pore cell, $L_{y}{ }^{\mathrm{p}}$, in the $y$ direction was $10 \sigma_{\mathrm{ff}}$ and $L_{z}{ }^{\mathrm{p}}$ in the $z$ direction was defined as the pore size $H$. Periodic boundary conditions were applied in the $x$ and $y$ directions. Similarly, the length of the silica cylindrical pore was set to be $L_{z}{ }^{\mathrm{p}}=70 \sigma_{\mathrm{ff}}$ and the FPF of $40 \sigma_{\mathrm{ff}}$ in length was located along the $z$ direction. The solid-fluid interaction potential by Eq. (11) in the FPF was linearly attenuated in the two PBFs of $15 \sigma_{\mathrm{ff}}$ in length along the $z$ direction. For both pore geometries, the size of the gas cell was set to be $L_{x}{ }^{g}=L_{y}{ }^{g}=L_{z}{ }^{g}=40 \sigma_{\mathrm{ff}}$ and periodic boundary conditions in the three directions were applied.

In analogy with the GC method mentioned in Sec. II C, two trial moves were performed in the OC method: displacement of a randomly selected particle and transfer of a particle selected at random from one cell to the other. The probabilities of the two trial moves were set to be equal. The acceptance rule, acc $(o \rightarrow n)$, for the trial displacement in the open pore cell and the gas cell is given by

$$
\operatorname{acc}(o \rightarrow n)=\min \left[1, \exp \left(-\frac{\Delta U}{k T}\right)\right],
$$

where $\Delta U$ is the potential energy change. The transfer of a particle from the open pore cell to the gas cell is accepted with

$$
\begin{aligned}
\operatorname{acc}(o \rightarrow n)= & \min \left[1, \exp \frac{N_{\mathrm{p}} V_{\mathrm{g}}}{\left(N_{\mathrm{g}}+1\right) V_{\mathrm{p}}}\right. \\
& \left.\times \exp \left(-\frac{\Delta U_{\mathrm{p}}+\Delta U_{\mathrm{g}}}{k T}\right)\right],
\end{aligned}
$$

where $N$ is the number of particles in a cell, and the subscripts $\mathrm{p}$ and $\mathrm{g}$ refer to the open pore cell and the gas cell, respectively. The acceptance rule for the transfer of a particle from the gas cell to the open pore cell is given by exchanging the subscripts in Eq. (15). The chemical potential of the system was obtained by using Eq. (12).

To assess the characteristics of our MC version of the OC method, we first generated an adsorption-desorption isotherm for a target pore by adding a given number of particles into the gas cell or removing them from the gas cell. The equilibrated configurations for the two cells were used as the initial configurations for the next simulation step, after the adding or removing of particles. The system was equilibrated for $1 \times 10^{4} \mathrm{MC}$ steps/particle, after which equilibrium properties of the system were collected for another $2 \times 10^{7}$ steps. Hereafter, this simulation procedure is referred to as the "FullTrace (FT) procedure."

As mentioned in Sec. II C, the characteristic of the open pore cell is that the adsorbed phase is steadily led to the thermodynamically stable state by forming concave menisci. Therefore, we adapted the following simulation procedure, which takes advantage of the feature of the open pore cell, to readily determine the equilibrium chemical potential. As a starting configuration, we used an equilibrated liquid phase in a pore with the same dimensions of the FPF of the target open pore cell. The configuration of particles was generated by the
GCMC method, namely, transferring it to the FPF portion of the open pore cell with nothing left in the gas cell, and beginning then the equilibration of the whole system. The equilibration was performed for $1 \times 10^{4} \mathrm{MC}$ steps/particle. Finally, another simulation run was conducted for $1 \times 10^{8} \mathrm{MC}$ steps, and the equilibrium properties were calculated from each of $2 \times 10^{7}$ MC steps to determine the standard error of the equilibrium chemical potential obtained by the OC method. In what follows, this simulation procedure is described as the "equilibrium shooting (ES) procedure."

\section{RESULTS AND DISCUSSION}

\section{A. Vapor-liquid coexistence by the OC method}

Figure 3 shows adsorption isotherms of the LJ nitrogen in the carbon slit pore of $H^{*}=10$ at $77 \mathrm{~K}$ obtained by the GCMC, GC, and OC methods, and snapshots [(a)-(d)] from the OC method. The overall density refers to the average density of the fluid $\rho=N / V$, where $N$ is the number of

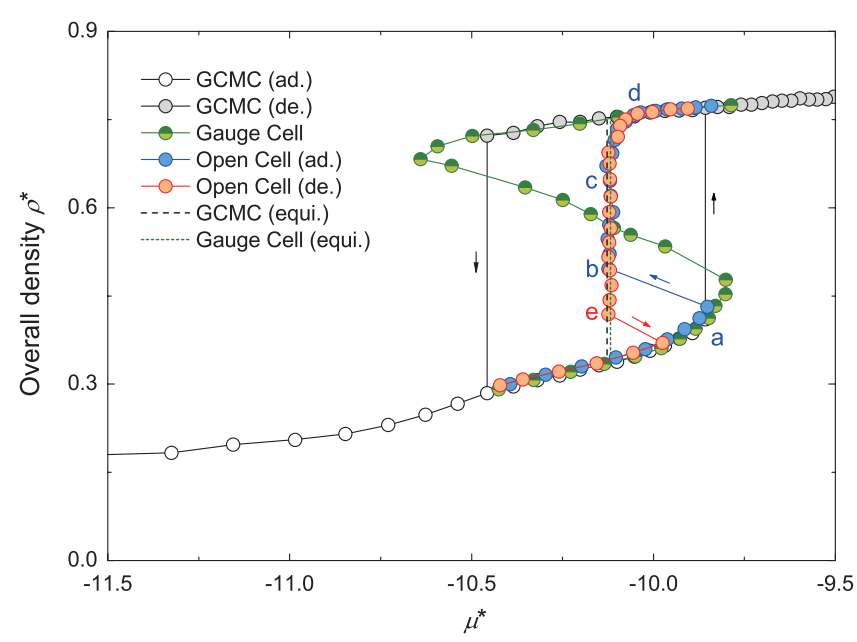

(a)

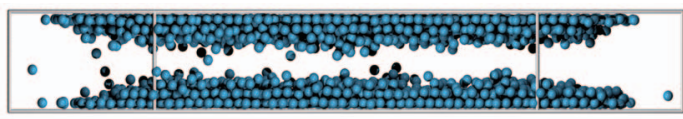

(b)

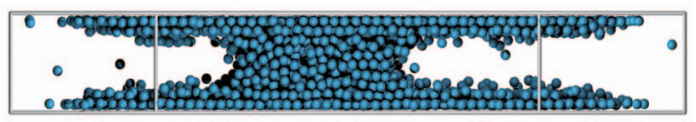

(c)

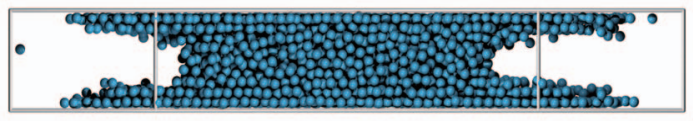

(d)

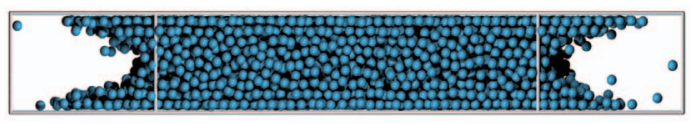

(e)

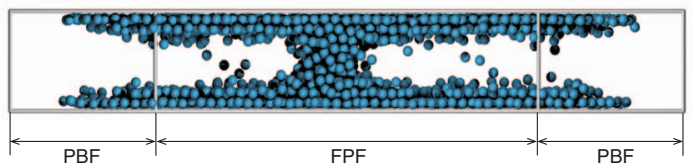

FIG. 3. Adsorption isotherms of the LJ nitrogen in the carbon slit pore of $H^{*}$ $=10$ at $77 \mathrm{~K}$ obtained by the GCMC, GC, and OC methods. In the case of the OC method, the overall density of the adsorbed phase is shown as the average density of particles in the whole FPF portion. Snapshots (a)-(d) correspond to points a-d on the adsorption and desorption branches obtained by the OC method. 
particles in the pore cell and $V$ is the volume of the pore cell for the GCMC and GC methods. In the case of the OC method, the overall density of the adsorbed phase is shown as the average density of particles in the whole FPF portion. For the GCMC isotherm, the equilibrium chemical potential, $\mu_{\mathrm{eq}}$, was determined by the PG method, while the $\mu_{\mathrm{eq}}$ value for the sigmoid GC adsorption isotherm was calculated by applying the Maxwell rule (Eq. (8)). We used the FT procedure of the OC method to generate the adsorption-desorption isotherm. The adsorption branch of the OC method goes through the metastable state in concurrence with the GCMC and GC isotherms, and at point $a$, spontaneous capillary condensation takes place. Once a liquid bridge with two concave menisci is formed in the open pore cell [snapshot (b)], it is clear that the system achieves its thermodynamically stable state, since the chemical potential of the system coincides with the $\mu_{\mathrm{eq}}$ values obtained by the PG and GC methods. As the number of particles in the total system increases, the liquid bridge grows along the $x$ direction, maintaining the same chemical potential [snapshot (c)]. However, after the two liquid menisci pass over the border of the FPF, the chemical potential of the system increases and deviates from the constant value [point $d$, see also snapshot (d)]. The OC desorption isotherm obtained by reducing the number of particles in the gas cell traces exactly the same path $(d-c-b)$ as the adsorption isotherm, however, the liquid bridge is still maintained at point $e$. The thickness of the liquid bridge is $\sim 8 \sigma_{\mathrm{ff}}$, and this may be the lower limit of the stable liquid bridge. These results suggest that, by using the OC method, we can obtain the equilibrium chemical potential with only a single simulation run, as long as the two liquid menisci are within the FPF. Moreover, the OC desorption isotherm directly indicates that the evaporation of condensate in an open pore system with a uniform pore width occurs at thermodynamic equilibrium.

Figure 4(a) shows the full density profile of the fluid in the open pore cell of the carbon slit pore of $H^{*}=10$ at $77 \mathrm{~K}$. The ES procedure of the open pore cell method was used to obtain the data. We also show the frequency plot of the particle insertion trial, whose outcome was a "success," in the $x, z$ plane of the open pore cell during $2 \times 10^{7}$ MC steps [Fig. 4(b)]. It is clear from Fig. 4(b) that the successful trials are found around the vapor-liquid interfaces. Note that exactly the same aspect was observed for the frequency plot of the successful trial of the particle removal from the open pore cell. These results suggest that the equilibrium properties of the gas cell are controlled by the shape of the concave meniscus. The shape of the liquid meniscus may depend on the setting of the PBF. We, therefore, removed the two PBFs to test the limiting case (the full potential was imposed throughout the pore cell), and re-equilibrated the system from the final configuration obtained by the ES procedure of the OC method. Figures 4(c) and 4(d) are the resulting full density profile of the fluid in the full potential pore cell and the frequency plot of the successful trial of the particle insertion, respectively. Apparently, the shape of the liquid meniscus in the full potential pore cell is different from that of the open pore cell with the PBF. However, we obtained the equilibrium chemical potential of $\mu_{\mathrm{eq}}{ }^{*}=-10.121$ for the full potential pore cell, which is in perfect agreement with the $\mu_{\mathrm{eq}}{ }^{*}$ value $(-10.121)$ (a)

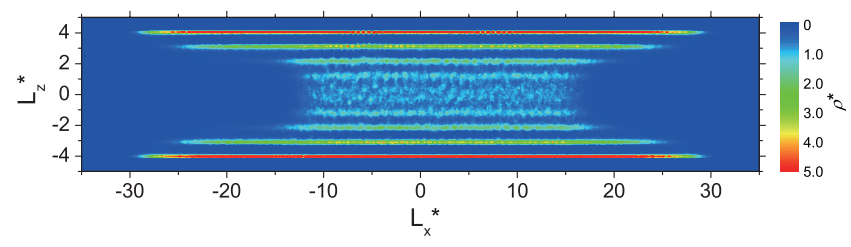

(b)

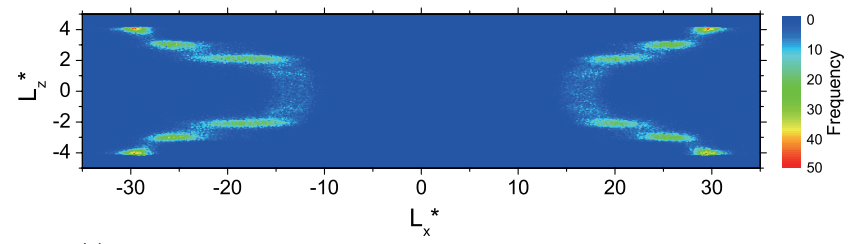

(c)

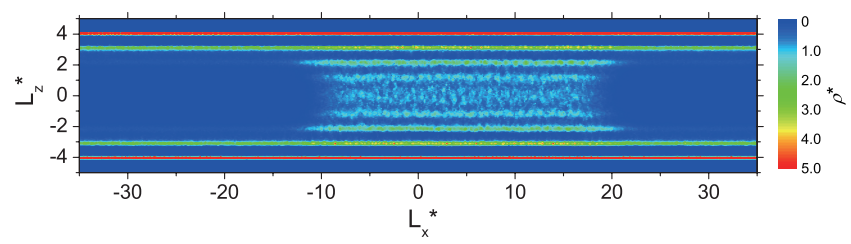

(d)

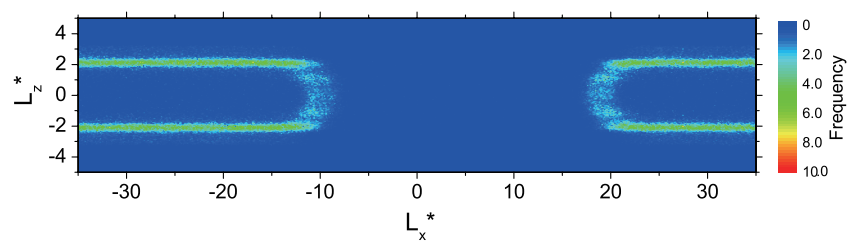

FIG. 4. Full density profiles of the LJ nitrogen for the carbon slit pore of $H^{*}$ $=10$ at $77 \mathrm{~K}$ : (a) the open pore cell and (c) the full potential pore cell without PBF. Frequency plots of the particle insertion trial in the $x$, $z$ plane, for which the outcome was a success: (b) the open pore cell and (d) the full potential pore cell without PBF.

from the regular OC method. In Fig. 5, we show the halfdensity points, $\rho_{\mathrm{h}}=1 / 2\left(\rho_{1}+\rho_{\mathrm{g}}\right)$, of the concave meniscus for the open pore cell and the full potential pore cell, where $\rho_{\mathrm{l}}$ and $\rho_{\mathrm{g}}$ are the liquid and gas densities at each $z$ position along the $x$ direction. The oscillation of the boundary of the concave meniscus is due to the layering of the adsorbed LJ nitrogen, as can be seen from the full density profile

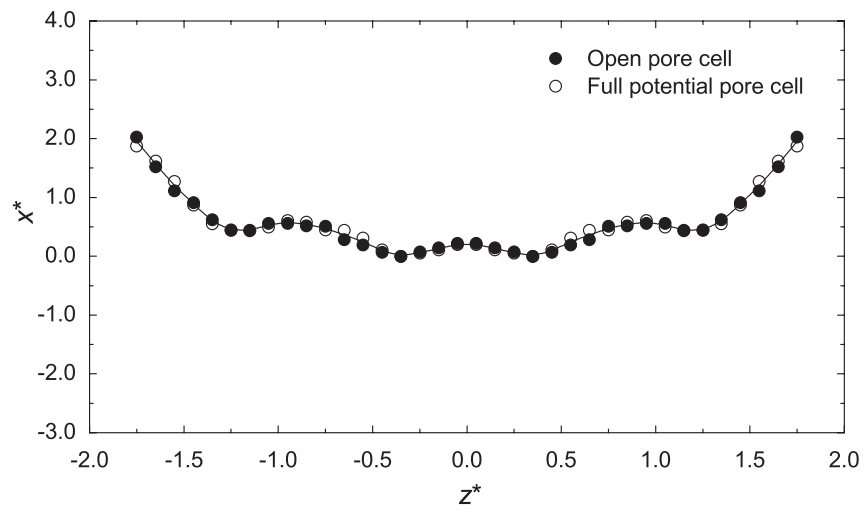

FIG. 5. Half-density points, $\rho_{\mathrm{h}}=1 / 2\left(\rho_{\mathrm{l}}+\rho_{\mathrm{g}}\right)$, of the concave meniscus for the open pore cell and the full potential pore cell, where $\rho_{\mathrm{l}}$ and $\rho_{\mathrm{g}}$ are the liquid and gas densities at each $z$ position along the $x$ direction. The positions of the boundary for both pore cells were shifted along the $x$ direction for comparison. 
[Figs. 4(a) and 4(c)]. By inspecting Fig. 5, we note that the boundary of the concave meniscus in the open pore cell perfectly coincides with that in the full potential pore cell. This suggests that the shape of the boundary at the concave meniscus formed in the pore is responsible for the equilibrium chemical potential. Moreover, in the case of the ES procedure of the OC method, the bottoms of the two concave menisci are within the FPF. This ensures that the solid-fluid interaction potentials exerted on the concave interfaces are equal to those for the full potential pore cell, and this is the reason why the shape of the boundary at the bottom of the concave meniscus and the resulting equilibrium chemical potential are equal between the two pore cells. It is also worth noting that the error in the equilibrium chemical potential from the regular OC method is \pm 0.006 , which is significantly smaller than that of the full potential pore cell $( \pm 0.022)$. This may be because of the existence of the wide thick multilayer regions in the full potential pore cell, which prevent effective sampling. Thus, this suggests that the open pore cell is useful for reducing the statistical errors in the equilibrium chemical potential.

\section{B. Comparison with the PG and GC methods}

Figures 6(a)-6(c) show adsorption isotherms of the LJ nitrogen in the carbon slit pores of $H^{*}=7,10$, and 13 at $77 \mathrm{~K}$ obtained by the GCMC and GC methods. The equilibrium chemical potentials determined for the respective slit pores by the PG, GC, and OC (ES procedure) methods are tabulated in Table II with the estimated errors, and also plotted in Figs. 6(a)-6(c) for comparison. As for the data from the OC method, the overall density of the adsorbed phase was calculated as the average density of particles in the whole FPF portion. The equilibrium chemical potentials obtained from the three different methods are in good agreement for all the slit pores, and the estimated errors of the mean are comparable in each case. This ensures that the OC method can be used for direct investigation of the equilibrium vapor-liquid coexistence controlled by the liquid concave meniscus, unlike the other two methods.

Adsorption isotherms of the LJ nitrogen in the silica cylindrical pores of $D^{*}=10,13$, and 16 at $77 \mathrm{~K}$ obtained by the GCMC and GC methods are shown in Figs. 7(a)7 (c) together with the equilibrium chemical potentials determined by the PG, GC, and OC (ES procedure) methods. The equilibrium chemical potentials are also tabulated in Table III with the estimated errors. Similar to what was observed for the carbon slit pores, the equilibrium chemical potentials obtained from the respective methods agree well. On the contrary, the errors in the equilibrium chemical potentials from the PG method are significantly larger than those from the GC method. As pointed out by Neimark and Vishnyakov, ${ }^{20}$ the errors of the PG method are attributed to the numerical instability of the thermodynamic integration along the path with a constant chemical potential. The errors of the OC method lie in between those of the other two methods. However, the equilibrium chemical potential obtained by the OC method is free of such numerical integration error and only contains the statistical error. In addition, the estimated er-
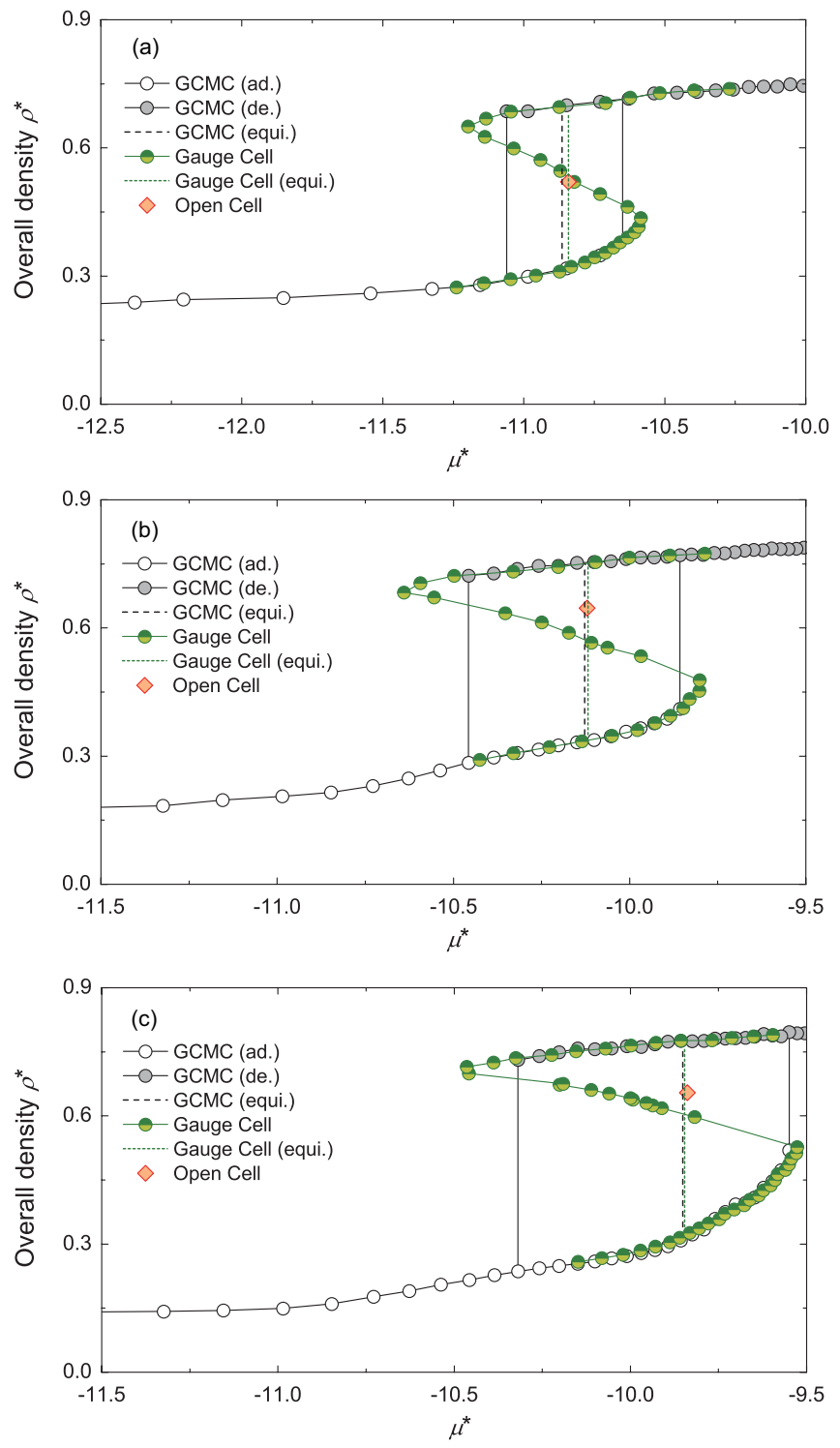

FIG. 6. Adsorption isotherms of the LJ nitrogen obtained by the GCMC and GC methods at $77 \mathrm{~K}$ for the carbon slit pores of (a) $H^{*}=7$, (b) $H^{*}=10$, and (c) $H^{*}=13$. The equilibrium chemical potentials determined for the respective slit pores by the PG, GC, and OC (ES procedure) methods are also shown for comparison. As for the data from the OC method, the overall density of the adsorbed phase was calculated as the average density of particles in the whole FPF portion.

rors in the equilibrium chemical potential for the OC method $(\leq \pm 0.008)$ are smaller than those of the points on the unstable states by the GC method, e.g., the estimated error in the chemical potential is \pm 0.010 at $\mu^{*}=-10.490$ and $\rho^{*}=0.602$ for the silica cylindrical pore of $D^{*}=10$. The relative stability

TABLE II. Reduced equilibrium chemical potentials $\left(\mu_{\mathrm{eq}} / \varepsilon_{\mathrm{ff}}\right)$ for the three slit pores obtained from the PG, GC, and OC (ES procedure) methods at $77.347 \mathrm{~K}^{\mathrm{a}}$

\begin{tabular}{lccc}
\hline \hline$H^{*}$ & PG & GC & OC \\
\hline 7 & $-10.856(5)$ & $-10.840(2)$ & $-10.842(8)$ \\
10 & $-10.128(3)$ & $-10.118(5)$ & $-10.121(6)$ \\
13 & $-9.850(2)$ & $-9.846(4)$ & $-9.849(4)$ \\
\hline \hline
\end{tabular}

${ }^{a}$ The numbers in parentheses are the estimated errors of the mean in the last decimal place, e.g., $-9.876(2)=-9.876 \pm 0.002$. 

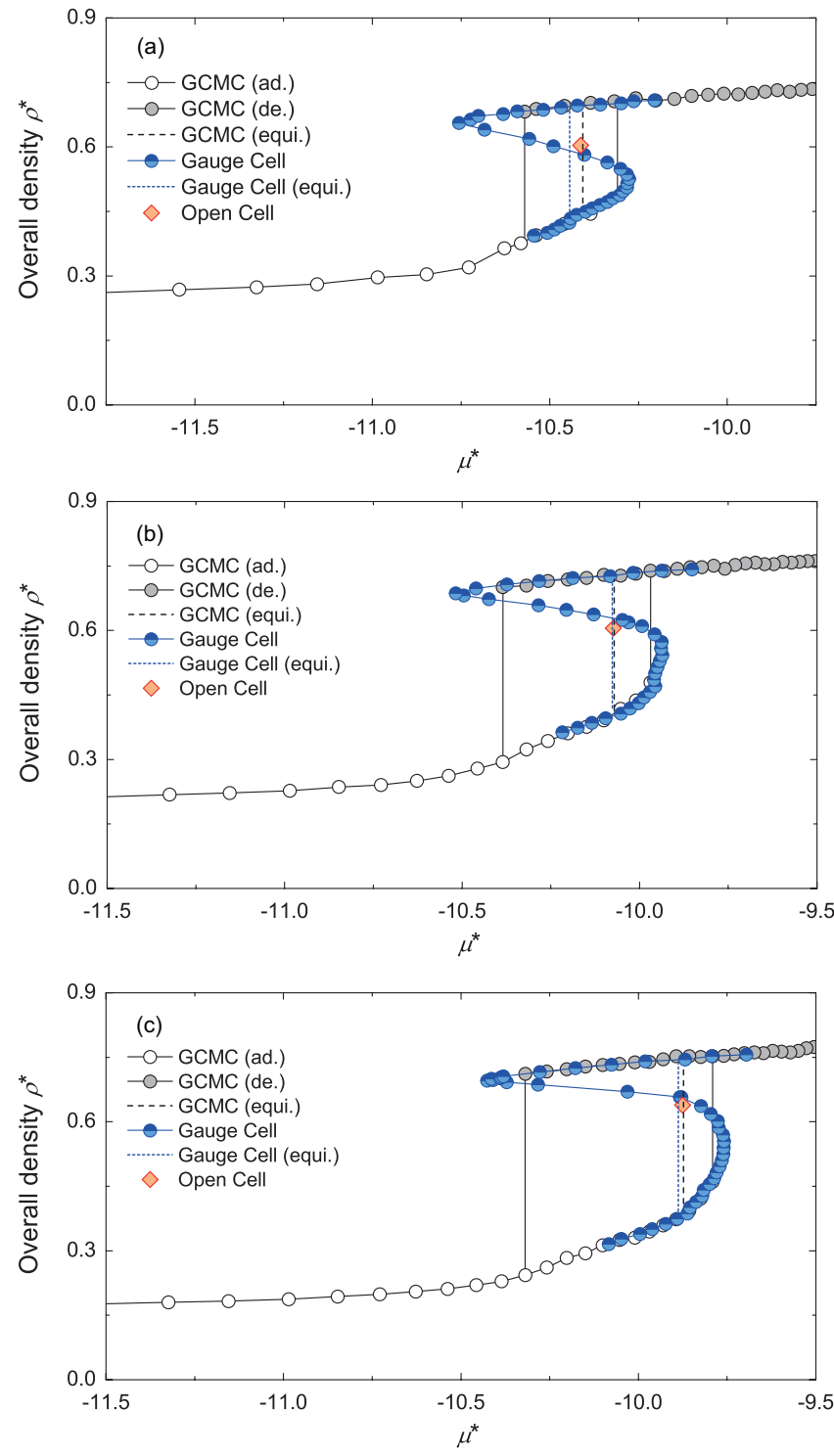

FIG. 7. Adsorption isotherms of the LJ nitrogen obtained by the GCMC and GC methods at $77 \mathrm{~K}$ for the silica cylindrical pores of (a) $D^{*}=10$, (b) $D^{*}=13$, and (c) $D^{*}=16$. The equilibrium chemical potentials determined for the respective cylindrical pores by the PG, GC, and OC (ES procedure) methods are also shown for comparison. As for the data from the OC method, the overall density of the adsorbed phase was calculated as the average density of particles in the whole FPF portion.

of the GC method may result from the fact that the obtained equilibrium chemical potential is an "average value" owing to the thermodynamic integration of the sigmoid adsorption isotherm.

TABLE III. Reduced equilibrium chemical potentials $\left(\mu_{\mathrm{eq}} / \varepsilon_{\mathrm{ff}}\right)$ for the three cylindrical pores obtained from the PG, GC, and OC (ES procedure) methods at $77.347 \mathrm{~K}^{\mathrm{a}}$

\begin{tabular}{lrrr}
\hline \hline$D^{*}$ & PG & GC & OC \\
\hline 10 & $-10.407(11)$ & $-10.444(2)$ & $-10.413(8)$ \\
13 & $-10.071(9)$ & $-10.077(2)$ & $-10.074(6)$ \\
16 & $-9.873(6)$ & $-9.888(1)$ & $-9.875(5)$ \\
\hline \hline
\end{tabular}

${ }^{a}$ The numbers in parentheses are the estimated errors of the mean in the last decimal place.
The total CPU time used for obtaining the equilibrium chemical potential for the carbon slit pore of $H^{*}=10$ with the OC method (ES procedure) was $1 \mathrm{~h}$ on an Intel HexaCore Xeon X5690 (3.47 GHz), where the simulation run was performed for $1 \times 10^{4} \mathrm{MC}$ steps/particle for equilibration and another run was conducted for $2 \times 10^{7} \mathrm{MC}$ steps for ensemble averaging. Conversely, the PG method required $40 \mathrm{~h}$ to obtain the equilibrium chemical potential for the carbon slit pore of $H^{*}=10$, where the simulation run was conducted for $5 \times 10^{7} \mathrm{MC}$ steps including the equilibration steps for each adsorption point, for a total of 173 adsorption points. The GC method needed $3.5 \mathrm{~h}$ for the same purpose, where the total number of adsorption datapoints was 28, and for each adsorption point, the system was equilibrated for $1 \times 10^{4} \mathrm{MC}$ steps/particle, after which the thermodynamic data were collected for another $2 \times 10^{7} \mathrm{MC}$ steps. However, in the case of the GC method, a certain amount of trial and error is required to determine the size of the gas cell in order to meet the condition of Eq. (13), and to determine the best way of adding particles to the system in order to obtain a continuous sigmoid adsorption isotherm. These facts indicate that the OC method shows a significant reduction in computational cost for obtaining the equilibrium chemical potential compared with the PG and GC methods.

\section{Application: Freezing in a confined system}

We also applied the OC method to determine the vaporliquid and vapor-solid coexistence curves in the carbon slit pore of $H^{*}=7.5$. The simulation was started from the temperature $T^{*}=k T / \varepsilon_{\mathrm{ff}}=0.76$, and the equilibrated configurations were used as the initial configurations for the following simulation step at a lower temperature. The volume of the gas cell was changed from $\left(40 \sigma_{\mathrm{ff}}\right)^{3}$ at $T^{*}=0.76$ to $\left(188 \sigma_{\mathrm{ff}}\right)^{3}$ at $T^{*}=0.54$ in order to maintain the number of particles in the gas cell (40-50 particles). The density of the condensate was evaluated as the average number density of particles in the central region of the open cell $\left(10 \sigma_{\mathrm{ff}} \times 10 \sigma_{\mathrm{ff}} \times H\right)$. Figures 8(a)-8(c) show the variations in density of the condensate versus temperature, the equilibrium vapor pressure, and the equilibrium chemical potential. The change in the equilibrium chemical potential shows an inflection point at $T^{*}=0.62$, which coincides with the sharp jump in the density of the condensate. The discrete changes are thus attributed to the liquid-solid phase transition of the condensate. Since the system automatically traces the vapor-liquid and vaporsolid coexistence curves, the observed freezing point corresponds to the triple point of the confined fluid. Such freezing behavior has been systematically investigated by the MD version of the OC method. ${ }^{21}$ However, the particle counting method employed in the MD version is of limited utility at low temperatures, because of the suppressed frequency of particle counting, which requires a long run of the MD simulation to obtain good statistics. On the contrary, our proposed method can overcome this difficulty by simply increasing the volume of the gas cell without increasing the number of simulation steps. It is worth noting that the chemical potential at the triple point of $T^{*}=0.62$ shows a small deviation from the vapor- 


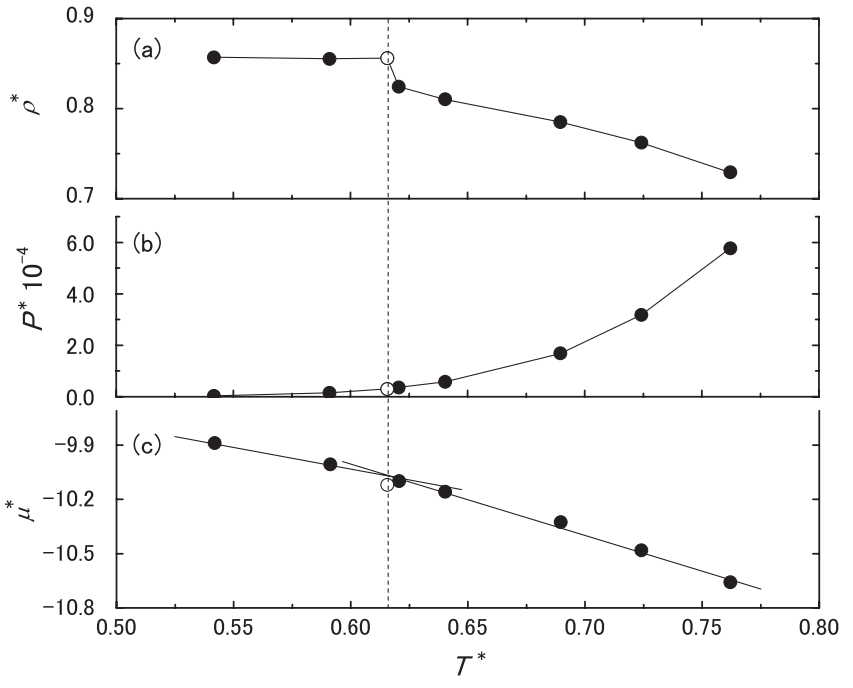

FIG. 8. Vapor-liquid and vapor-solid coexistences of the confined LJ nitrogen in the carbon slit pore of $H^{*}=7.5$ obtained by the OC method showing (a) the variation in overall density in the central region of the open cell $\left(10 \sigma_{\mathrm{ff}}\right.$ $\times 10 \sigma_{\mathrm{ff}} \times H$ ), (b) the change in the equilibrium vapor pressure, and (c) the change in the equilibrium chemical potential. The dashed line and open circles indicate the triple point of the confined LJ nitrogen.

liquid and vapor-solid coexistence lines. This may be due to the so-called surface melting of the confined LJ particles at the concave meniscus, and this finding suggests that our OC method can be applied to investigate such a phenomenon.

\section{CONCLUSIONS}

We developed the OC method to determine the phase equilibria in nanopores. The method employs the open pore cell with the PBF and combines it with the gas cell to measure the chemical potential of the system in analogy with the GC method. We compared the equilibrium chemical potentials of confined nitrogen in carbon slit pores and silica cylindrical pores at $77 \mathrm{~K}$ with those of the PG method and the GC method. The equilibrium chemical potentials obtained from the three methods are in good agreement for all the pore models employed, and this suggests that the OC method can be used to directly investigate the equilibrium vapor-liquid coexistence controlled by the liquid concave meniscus.

The OC method enables one to estimate the equilibrium chemical potential of the confined fluid with a single simulation, unlike the PG method and the GC method. The low computational cost to evaluate the equilibrium chemical potential will allow us to construct kernels composed of adsorption isotherms by the GCMC method, which are more precise than those obtained by nonlocal density functional theory to calculate the pore size distributions of porous materials.

We also demonstrated the excellent properties of the OC method for obtaining a vapor-solid coexistence curve in a narrow pore in which the equilibrium vapor pressure is extremely low, and for locating the triple point of a confined fluid. In the MD version of the open cell method, the computational cost becomes large at low temperatures and associated low pressures. However, our proposed method can overcome this disadvantage by simply adjusting the size of the gas cell. This suggests that the OC method would be particularly useful in the investigation of solid-solid phase transitions of confined fluids such as $\mathrm{H}_{2} \mathrm{O}, \mathrm{N}_{2}$, and $\mathrm{O}_{2}$, which exhibit a variety of solid-solid phase transitions in the bulk. The combination of the OC method and the parallel tempering technique would offer a powerful tool for exploring such solid-solid phase transitions in confined systems.

\section{ACKNOWLEDGMENTS}

The authors are grateful to Dr. Nakai, Mr. Iegami, Mr. Funahashi, Mr. Matsui, and Mr. Kikyotani for their assistance in this work. This work was financially supported by the "Development of Advanced Measurement and Analysis Systems" Project of the Japan Science and Technology Agency (JST) and a Grant-in-Aid for Scientific Research (B) 24360318 from MEXT.

${ }^{1}$ B. Widom, J. Stat. Phys. 19, 563 (1978).

${ }^{2}$ G. S. Heffelfinger, F. Vanswol, and K. E. Gubbins, Mol. Phys. 61, 1381 (1987).

${ }^{3}$ J. F. Knight and P. A. Monson, J. Chem. Phys. 84, 1909 (1986).

${ }^{4}$ J. F. Knight and P. A. Monson, Mol. Phys. 60, 921 (1987).

${ }^{5}$ B. K. Peterson, K. E. Gubbins, G. S. Heffelfinger, U. M. B. Marconi, and F. Vanswol, J. Chem. Phys. 88, 6487 (1988).

${ }^{6}$ G. S. Heffelfinger, F. Vanswol, and K. E. Gubbins, J. Chem. Phys. 89, 5202 (1988).

${ }^{7}$ J. G. Powles, S. E. Baker, and W. A. B. Evans, J. Chem. Phys. 101, 4098 (1994).

${ }^{8}$ S. Tripathi and W. G. Chapman, Mol. Phys. 101, 1199 (2003).

${ }^{9}$ D. Frenkel and B. Smit, Understanding Molecular Simulation: From Algorithms to Applications (Academic, New York, 2002).

${ }^{10}$ B. K. Peterson and K. E. Gubbins, Mol. Phys. 62, 215 (1987).

${ }^{11}$ S. Jiang, C. L. Rhykerd, and K. E. Gubbins, Mol. Phys. 79, 373 (1993).

${ }^{12}$ J. Puibasset, J. Phys. Chem. B 109, 480 (2005).

${ }^{13}$ A. Papadopoulou, F. van Swol, U. M. B. Marconi, J. Chem. Phys. 97, 6942 (1992).

${ }^{14}$ A. Z. Panagiotopoulos, Mol. Phys. 62, 701 (1987).

${ }^{15}$ S. C. McGrother and K. E. Gubbins, Mol. Phys. 97, 955 (1999).

${ }^{16}$ I. Brovchenko, A. Geiger, and A. Oleinikova, Phys. Chem. Chem. Phys. 3, 1567 (2001).

${ }^{17}$ A. Vishnyakov, E. M. Piotrovskaya, E. N. Brodskaya, E. V. Votyakov, and Yu. K. Tovbin, Langmuir 17, 4451 (2001).

${ }^{18}$ I. Brovchenko, A. Geiger, and A. Oleinikova, J. Chem. Phys. 120, 1958 (2004).

${ }^{19}$ I. Brovchenko, A. Geiger, and A. Oleinikova, J. Phys.: Condens. Matter 16, S5345 (2004).

${ }^{20}$ A. V. Neimark and A. Vishnyakov, Phys. Rev. E 62, 4611 (2000).

${ }^{21}$ M. Miyahara, T. Yoshioka, and M. Okazaki, J. Chem. Phys. 106, 8124 (1997).

${ }^{22}$ M. Miyahara, H. Kanda, M. Shibao, and K. Higashitani, J. Chem. Phys. 112, 9909 (2000).

${ }^{23}$ H. Kanda, M. Miyahara, and K. Higashitani, J. Chem. Phys. 120, 6173 (2004).

${ }^{24}$ H. Kanda, M. Miyahara, and K. Higashitani, Adsorption 11, 295 (2005).

${ }^{25} \mathrm{H}$. Kanda and M. Miyahara, Adsorption 13, 191 (2007).

${ }^{26}$ H. Kanda and M. Miyahara, J. Chem. Phys. 126, 054703 (2007).

${ }^{27}$ T. Yoshioka, M. Miyahara, and M. Okazaki, J. Chem. Eng. Jpn. 30, 274 (1997).

${ }^{28}$ M. Miyahara, H. Kanda, T. Yoshioka, and M. Okazaki, Langmuir 16, 4293 (2000).

${ }^{29}$ H. Kanda, M. Miyahara, and K. Higashitani, Langmuir 16, 6064 (2000).

${ }^{30}$ H. Kanda, M. Miyahara, T. Yoshioka, and M. Okazaki, Langmuir 16, 6622 (2000).

${ }^{31}$ J. K. Johnson, J. A. Zollweg, and K. E. Gubbins, Mol. Phys. 78, 591 (1993).

${ }^{32}$ A. V. Neimark and A. Vishnyakov, J. Chem. Phys. 122, 234108 (2005).

${ }^{33}$ A. Vishnyakov and A. V. Neimark, J. Phys. Chem. B 105, 7009 (2001).

${ }^{34}$ M. Jorge and N. A. Seaton, Mol. Phys. 100, 3803 (2002).

${ }^{35}$ M. Jorge, C. Schumacher, and N. A. Seaton, Langmuir 18, 9296 (2002). 
${ }^{36}$ A. Vishnyakov and A. V. Neimark, J. Chem. Phys. 119, 9755 (2003).

${ }^{37}$ A. Vishnyakov and A. V. Neimark, Langmuir 19, 3240 (2003).

${ }^{38}$ J. W. Jiang, S. I. Sandler, and B. Smit, Nano Lett. 4, 241 (2004).

${ }^{39}$ A. V. Neimark and A. Vishnyakov, J. Chem. Phys. 122, 054707 (2005).

${ }^{40}$ P. Kowalczyk, R. Holyst, H. Tanaka, and K. Kaneko, J. Phys. Chem. B 109, 14659 (2005).

${ }^{41}$ A. V. Neimark and A. Vishnyakov, J. Phys. Chem. B 110, 9403 (2006).

${ }^{42}$ J. P. B. Mota and I. Esteves, Adsorption 13, 21 (2007).

${ }^{43}$ A. Vishnyakov and A. V. Neimark, J. Chem. Phys. 130, 224103 (2009).

${ }^{44}$ C. J. Rasmussen, A. Vishnyakov, and A. V. Neimark, J. Chem. Phys. 135, 214109 (2011).
${ }^{45}$ Z. J. Liu, L. Herrera, V. T. Nguyen, D. D. Do, and D. Nicholson, Mol. Simul. 37, 932 (2011).

${ }^{46}$ Z. J. Liu, D. D. Do, and D. Nicholson, Mol. Simul. 38, 189 (2011).

${ }^{47}$ B. Coasne, A. Galarneau, F. Di Renzo, and R. J. M. Pellenq, J. Phys. Chem. C 111, 15759 (2007).

${ }^{48}$ B. Coasne, A. Galarneau, F. Di Renzo, and R. J. M. Pellenq, Langmuir 26, 10872 (2010).

${ }^{49}$ P. I. Ravikovitch, A. Vishnyakov, and A. V. Neimark, Phys. Rev. E 64, 011602 (2001).

${ }^{50}$ G. J. Tjatjopoulos, D. L. Feke, and J. A. Mann, Jr., J. Phys. Chem. 92, 4006 (1988). 